\title{
Random Generalized Bi-linear Mixed Variational-like Inequality for Random Fuzzy Mappings
}

\author{
Hongxia Dai
}

Department of Economic Mathematics, Southwestern University of Finance and Economics, Chengdu 610074,

\author{
P.R.China
}

\begin{abstract}
In this paper, we introduce and study a new class of random generalized bi-linear mixed variational-like inequality for random fuzzy mappings. By using the minimax inequality and extending auxiliary principle, we prove an existence and uniqueness theorem of the solution for the random generalized bi-linear mixed variational-like inequality.
\end{abstract}

Keywords: Random generalized bi-mixed variational-like inequality, Minimax inequality, auxiliary principle

\section{Introduction}

It is well known that variational inequality theories are very effective and powerful tools for studying a wide class of linear and nonlinear problems arising in many diverse fields of pure and applied sciences such as mechanics, physics, optimization and control, nonlinear programming, economics and transportation equilibrium, and engineering sciences, etc. In recent years, classical variational inequality theories have been generalized and applied in various directions, the readers refer to [1]-[3] and the references therein. A useful and important generalization of variational inequalities is the mixed variational-like inequalities, which have potential and significant applications in optimization theory [4,5], structural analysis [6], and economics [7,8]. It is noted that there are many effective numerical methods for finding approximate solutions of various variational inequalities. Among these methods, the projection method and its variant forms is the most effective numerical technique. However, the projection type technique cannot be used to study mixed variational-like inequalities, since it is not possible to find the projection of the solution. These facts motivated Glowinski et al. [7] to suggest another technique, which does not depend on the projection. The technique is called the auxiliary principle technique. Very recently, Huang et al. [9] and Ding [10] extend the auxiliary principle technique to study generalized nonlinear mixed variational-like inequalities.

On the other hand, in 1989, Chang and Zhu [11] introduced the concept of variational inequality for fuzzy mappings, which was extended by Lassonde [8], Shih and Tan [12]. Recently, the random variational inequalities have been introduced and studied (see [13, 14,15]-[17]).

Inspired and motivated by recent works $[18,19,14,16,17]$, we introduce and study a class of random generalized bi-linear mixed variational-like inequality for random fuzzy mapping. By using the minimax inequality and extending auxiliary principle, we prove the existence and uniqueness theorem of the solution for the random generalized bi-linear mixed variational-like inequality. Our results improve and generalize many known corresponding results presented in [10,13,20,14,9].

\section{Preliminaries}

Throughout this paper, let $H$ be a real Hilbert space with norm and inner product denoted by $\|\cdot\|$ and $\langle\cdot \cdot\rangle$, respectively, and $D$ be a nonempty closed convex subset of $H$. We denote by $2^{H}$ and $C B(H)$ the families of all the nonempty subsets and the families of the nonempty bounded closed subsets of $H$, respectively. $H(\cdot, \cdot)$ represents the Hausdorff metric on $C B(H)$.

Let $(\Omega, \Sigma)$ be a measurable space, where $\Omega$ is a set and $\Sigma$ is $\sigma$-algebra of subsets of $\Omega$. We denote by $\beta(H)$ the class of Borel $\sigma$ - fields in $H$.

Definition 2.1. A mapping $f: \Omega \rightarrow H$ is said to be measurable if for any $C \in \beta(H)$ and

$$
f^{-1}(C)=\{t \in \Omega: f(t) \in C\} \in \Sigma \text {. }
$$

Definition 2.2. A mapping $f: \Omega \times H \rightarrow H$ is called a random operator if for any $w \in H, f(t, w)=w(t)$ is measurable. A random operator $f: \Omega \times H \rightarrow H$ is said to be continuous if for any $t \in \Omega$, the mapping $f(t, \cdot): H \rightarrow H$ is continuous.

Definition 2.3. A multivalued mapping $A: \Omega \rightarrow C B(H)$ is said to be measurable if for any $C \in \beta(H)$ and

$$
A^{-1}(C)=\{t \in \Omega: A(t) \cap C \neq \Phi\} \in \Sigma .
$$

Definition 2.4. A mapping $u: \Omega \rightarrow H$ is called a measurable selection of the multivalued measurable mapping $A: \Omega \rightarrow C B(H)$ if $u$ is a measurable mapping and $t \in \Omega, u(t) \in A(t)$.

Definition 2.5. A mapping $T: \Omega \times H \rightarrow C B(H)$ is called a random multivalued mapping if for any $w \in H, T(\cdot, w)$ is measurable. A random multivalued mapping $T: \Omega \times H \rightarrow C B(H)$ is said to be $H$ - continuous if for any $t \in \Omega, T(t, \cdot)$ is continuous in the Hausdorff metric.

Let $F(H)$ be a collection of fuzzy sets over 
$H$. A mapping $\widetilde{F}$ from $\Omega$ into $F(H)$ is called a fuzzy mapping. If $\widetilde{F}$ is a fuzzy mapping on $H$, for any $t \in \Omega, \tilde{F}(t)$ (denote it by $\tilde{F}_{t}$ in the sequel) is a fuzzy set on $H$ and $\tilde{F}_{t}(z)$ is the membership function of $z$ in $\tilde{F}_{t}$. Let $M \in F(H)$, $q \in[0,1]$, then the set

$$
(M)_{q}=\{u \in H: M(u) \geq q\}
$$

is called a $q$-cut set of $M$.

Definition 2.6. A fuzzy mapping $\tilde{F}: \Omega \rightarrow F(H)$ is called measurable if for any $a \in[0,1],(\tilde{F}(\cdot))_{a}$ : $\Omega \rightarrow 2^{H}$ is a measurable multivalued mapping.

Definition 2.7. A fuzzy mapping $\tilde{F}: \Omega \times H \rightarrow$ $F(H)$ is called a random fuzzy mapping if for any $w \in H, \quad \tilde{F}(\cdot, w): \Omega \rightarrow F(H)$ is a measurable fuzzy mapping.

Clearly, the random fuzzy mapping includes multi-valued mappings, random multivalued mappings and fuzzy mappings as the special cases.

Let $\tilde{A}, \tilde{T}: \Omega \times H \rightarrow F(H)$ be two random fuzzy mappings satisfying the following condition (I): if there exist two mappings $a, c: H \rightarrow[0,1]$ such that

$$
\begin{aligned}
\forall(t, w) \in \Omega \times H,\left(\tilde{A}_{t, w}\right)_{a(w)} & \in C B(H), \\
\left(\tilde{T}_{t, w}\right)_{c(w)} & \in C B(H) .
\end{aligned}
$$

By using the random fuzzy mappings $\tilde{A}$ and $\tilde{T}$, we can define two random multi-valued mappings $A$ and $T$ as follows:

$$
\begin{aligned}
& \forall(t, w) \in \Omega \times H \\
& A: \Omega \times H \rightarrow C B(H),(t, w) \rightarrow\left(\tilde{A}_{t, w}\right)_{a(w)}, \\
& T: \Omega \times H \rightarrow C B(H),(t, w) \rightarrow\left(T_{t, w}\right)_{c(w)} .
\end{aligned}
$$

So $A$ and $T$ are called the random multi-valued mappings induced by the random fuzzy mappings $\tilde{A}$ and $\tilde{T}$, respectively.

Given mappings $a, c: H \rightarrow[0,1]$, the random fuzzy mappings $\tilde{A}, \tilde{T}: \Omega \times H \rightarrow F(H)$ satisfy the condition (I). Let $N, \eta: H \times H \rightarrow H$ be two mappings. Let $b: H \times H \rightarrow(-\infty,+\infty]$ be a real-valued function. We shall study the following problem: Find measurable mappings $u, x, y: \Omega$ $\rightarrow H$ such that

$$
\tilde{A}_{t, u(t)}(x(t)) \geq a(u(t)), \tilde{T}_{t, u(t)}(y(t)) \geq c(u(t))
$$

and

$$
\begin{aligned}
& <N(x(t), y(t)), \eta(v, u(t))> \\
& \quad+b(u(t), v)-b(u(t), u(t)) \geq 0,
\end{aligned}
$$

for all $t \in \Omega$ and $v \in H$, where the function $b(\cdot, \cdot)$ is nondifferential and satisfies the following conditions:

(i) for any $w, v \in H, b(w, v)$ is line in the first argument;

(ii) for each $w \in H, b(w, \cdot)$ is a convex function;

(iii) for any $w, v \in H, b(w, v)$ is bounded, that is, there exists a constant $\gamma>0$ such that

(iv) for all $w, v, z \in H$ $b(w, v) \leq \gamma\|w\| \cdot\|v\|$

\section{Remark 2.1.}

$$
b(w, v)-b(w, z) \leq b(w, v-z) \text {. }
$$

(1) for any $w, v \in H, b(-w, v)=-b(w, v)$ and $b(-w, v) \leq \gamma\|w\| \cdot\|v\|$ hold from condition (i) and (iii), respectively. So $|b(w, v)| \leq \gamma\|w\| \cdot\|v\|$.

(2) for any $w, v, z \in H,|b(w, v)-b(w, z)| \leq$ $\gamma\|w\| \cdot\|v-z\|$ from condition (ii) and (iv). So $b(w, v)$ is continuous with respect to second argument.

Inequality (2.1) is called random generalized bi-linear mixed variational-like inequality for random fuzzy mappings. The set of measurable mappings $(u, x, y)$ is called a random solution of the random generalized bi-linear mixed variational-like inequality.

Special cases:

(1) If $N(x(t), y(t))=P(t, x(t))-F(t, y(t))$, $\eta(v, u(t))=v-g(t, u(t))$, where $F=f-g$, $P, f, g: \Omega \times H \rightarrow H$, and $b(u, v)=\phi(v)$ for all $u, v \in H$, the problem (2.1) reduces to the following random generalized nonlinear mixed variational inclusions for random fuzzy mappings: Find measurable mappings $u, x, y, w: \Omega \rightarrow H$, such that for all $t \in \Omega, u(t) \in H, \tilde{A}_{t, u(t)}(x(t)) \geq$ $a(u(t)), \tilde{T}_{t, u(t)}(y(t)) \geq c(u(t)), S_{t, u(t)}(w(t)) \geq$ $d(u(t)), g(t, w(t)) \cap \operatorname{dom}(\partial \phi) \neq \Phi$ and $<P(t, x(t))-\{f(t, y(t))-g(t, w(t))\}, v-g(t, w(t))>$

$$
\geq \phi(g(t, w(t)))-\phi(v), \quad \forall v \in H .
$$

The problem(2.2) was studied by Ahmad and Bazan [13].

(2) If $N(x(t), y(t))=f(t, x(t))-p(t, y(t))$, $\eta(v, u(t))=v-g(t, u(t)) \quad$ and $\quad b(u, v)=\phi(v)$ for all $u, v \in H$, the problem (2.1) reduces to the following random generalized nonlinear variational inclusions for random fuzzy mappings: Find measurable mappings $u, x, y: \Omega \rightarrow H$, such that for all $t \in \Omega, u(t) \in H, \tilde{A}_{t, u(t)}(x(t)) \geq a(u(t))$, $\tilde{T}_{t, u(t)}(y(t)) \geq c(u(t))$ and

$$
<f(t, x(t))-p(t, y(t)), v-g(t, u(t))>
$$


$\geq \phi(g(t, u(t)))-\phi(v), \quad \forall v \in H$.

The problem(2.3) was studied by Huang [14].

(3) If in Banach space, let $\tilde{A}, \tilde{T}: \Omega \times D$ $\rightarrow B^{\square}$ be measurable mappings, $N(\cdot, \cdot)=T(\cdot)-$ $A(\cdot)$ and the real-valued function $b(u, v)=f(v)$ for all $u, v \in D$, then the problem (2.1) reduces to the following random mixed variational-like inequality problem: $\forall v \in B$

$$
\begin{aligned}
& <T(t, u(t))-A(t, u(t)), \eta(v, u(t))> \\
\leq & f(u(t))-f(v) .
\end{aligned}
$$

The problem (2.4) was considered by Ding [20].

(4) If in reflexive Banach spaces, let $\tilde{A}, \tilde{T}: D \rightarrow B^{\square} \quad$ and $\quad \eta: D \times D \rightarrow B \quad$ be mappings, then the problem (2.1) reduces to the following nonlinear mixed variational-like inequality: for a given $w^{\square} \in B^{\square}$, find $u \in D$ such that $<N(T u, A u)-w, \eta(v, u)>+b(u, v)-b(u, u)$

$$
\geq 0, \quad \forall v \in B \text {. }
$$

The problem (2.5) was considered by Ding [10].

It is noted that the problems $(2.2)-(2.5)$ are special cases of the problem (2.1). In brief, problem (2.1) is the most general and unifying one, which is also one of the main motivations of this paper.

Definition 2.8. Let $D$ be a nonempty closed convex subset of $H$, a mapping $\eta: D \times D \rightarrow D$ is called $\sigma$-Lipschitz continuous, if there exists a constant $\sigma>0$ such that

$$
\|\eta(u, v)\| \leq \sigma\|u-v\|, \quad \forall u, v \in D .
$$

Definition 2.9. Let $D$ be a nonempty closed convex subset of $H$, let $\eta: D \times D \rightarrow D$ and $N(\cdot, \cdot): D$ $\times D \rightarrow D$ be two mappings.

(1) $N(\cdot, \cdot)$ is said to be Lipschitz continuous in first argument, if there exists a constant $r>0$ such that

$\|N(u, \cdot)-N(v, \cdot)\| \leq r\|u-v\|, \quad \forall u, v \in D$.

(2) $N(\cdot, \cdot)$ is said to $\eta$-strongly monotone in first argument with respect to the random multivalued mapping $A$, if there exists a constant $\delta>0$ such that for any $t \in \Omega$,

$$
\begin{gathered}
<N\left(x_{1}, \cdot\right)-N\left(x_{2}, \cdot\right), \eta\left(u_{1}, u_{2}\right)>\geq \delta\left\|u_{1}-u_{2}\right\|^{2}, \\
\forall u_{1}, u_{2} \in H, x_{1} \in A\left(t, u_{1}\right), x_{2} \in A\left(t, u_{2}\right) .
\end{gathered}
$$

Similarly, we can define Lipschitz continuity and the $\eta$-strongly monotonicity of $N(\cdot, \cdot)$ in second argument with respect to the random multi-valued mappings $T$.

Definition 2.10. Let $A, T: \Omega \times H \rightarrow C B(H)$ be two random multi-valued mappings induced by the random fuzzy mappings $\tilde{A}$ and $\tilde{T}$, respectively, and $\eta: D \times D \rightarrow D$ be mapping. The mappings $u \rightarrow N(x(t), y(t))$ and $\eta$ are said to have $0-$ diagonally concave relation, if for any $t \in \Omega$, the function $\phi: \Omega \times D \times D \rightarrow(-\infty,+\infty]$ defined by

$$
\phi(t, v, u)=\langle N(x(t), y(t)), \eta(u, v)\rangle
$$

has 0 -diagonally concave in $v$, where $x(t) \in$ $A(t, u), y(t) \in T(t, u)$, i.e., for any $t \in \Omega$, any finite set $\left\{v_{1}, v_{2}, \ldots, v_{m}\right\} \subset D$ and $u=\sum_{i=1}^{m} \lambda_{i} v_{i}$ $\left(\lambda_{i} \geq 0, \sum_{i=1}^{m} \lambda_{i}=1\right), \sum_{i=1}^{m} \lambda_{i} \phi\left(t, v_{i}, u\right) \leq 0$.

\section{Existence uniqueness theorem}

At first, we give the following Lemmas.

Lemma 3.1. [21] Let $T: \Omega \times H \rightarrow C B(H)$ be a $\hat{H}$ - continuous random multivalued mapping, then for measurable mapping $u: \Omega \rightarrow H$, the multivalued mapping $T(\cdot, u(\cdot)): \Omega \rightarrow C B(H)$ is measurable.

Lemma 3.2. [20] Let $(\Omega, \Sigma)$ be a measurable space, and $D$ be a nonempty convex subset of a topologi- cal vector space. Let $\varphi: \Omega \times D \times D \rightarrow[-\infty,+\infty]$ be a real-valued function such that

(1) for each $(v, u) \in D \times D, t \rightarrow \varphi(t, v, u)$ is measurable mapping;

(2) for each $(t, v) \in \Omega \times D, u \rightarrow \varphi(t, v, u)$ is continuous on each nonempty compact subset of $D$;

(3) for each $(t, u) \in \Omega \times D, \quad v \rightarrow \varphi(t, v, u)$ is lower semicontinuous on each nonempty compact subset of $D$;

(4) for each $t \in \Omega$, each nonempty finite set $\left\{v_{1}, v_{2}, \ldots, v_{m}\right\} \subset D$ and for each $u=\sum_{i=1}^{m} \lambda_{i} v_{i}$ $\left(\lambda_{i} \geq 0\right.$, and $\left.\sum_{i=1}^{m} \lambda_{i}=1\right), \min _{1 \leq i \leq m} \varphi\left(t, v_{i}, u\right) \leq 0$;

(5) for each $t \in \Omega$, there exists a nonempty compact convex subset $D_{0}$ of $D$ and a nonempty compact subset $K$ of $D$ such that for each $u \in D \backslash K$, there is a $v \in \operatorname{co}\left(D_{0} \cup\{u\}\right)$ with $\varphi(t, v, u)>0$.

Then there exists a measurable mapping $u: \Omega \rightarrow D$ such that $\varphi(t, v, u(t)) \leq 0$ for all $v \in D$ and $t \in \Omega$.

Now we now state the main result of this paper. Theorem 3.1. Let $(\Omega, \Sigma)$ be a measurable space, and $D$ be a nonempty convex subset of $H$. Let random fuzzy mappings $\tilde{A}, \tilde{T}: \Omega \times H \rightarrow F(H)$ 
satisfy the condition (I), and $A$ and $T$ be the random multi-valued mappings induced by the random fuzzy mappings $\tilde{A}$ and $\tilde{T}$, respectively. Let $N, \eta: D \times D \rightarrow D$ be two mappings. Let $b: D \times D \rightarrow(-\infty,+\infty]$ be a real-valued function such that

(1) for each $t \in \Omega$, the mapping $A(t, \cdot), T(t$, $\cdot)$ is $\hat{H}$ - continuous with constant $0<\lambda_{1}, \lambda_{2} \leq 1$, respectively;

(2) the mapping $\eta$ is Lipschitz continuous with constant $\sigma>0$; the mapping $\eta(u, v)$ is continuous in first argument and semicontinuous in second argument, and for all $u, v \in D, \eta(u, v)=$ $-\eta(v, u)$;

(3) the mapping $N(\cdot, \cdot)$ is Lipschitz continuous and $\eta$-strongly monotone with respect to the random multi-valued mapping $A$ in first argument with constant $k_{11}>0$ and $k_{21}>0$ respectively. $N(\cdot, \cdot)$ is Lipschitz continuous and $\eta$ - strongly monotone with respect to the random multi-valued mapping $T$ in second argument with constant $k_{12}>0$ and $k_{22}>0$ respectively, too;

(4) for each $t \in \Omega$, the mappings $u \rightarrow N$ ( $x(t), y(t))$ and $\eta$ have the 0 -diagonally concave relation;

(5) the function $b(\cdot, \cdot)$ satisfies conditions (i) - (iv) where $\gamma \in\left(0, k_{21}+k_{22}\right)$.

Then the problem (2.1) has a unique random solution $\hat{u}(t) \in D, \hat{x}(t) \in A(t, \hat{u}(t)), \hat{y}(t) \in T(t, \hat{u}(t))$, i.e.

$$
\begin{aligned}
& \langle N(\hat{x}(t), \hat{y}(t)), \eta(v, \hat{u}(t))\rangle+b(\hat{u}(t), v)- \\
& b(\hat{u}(t), \hat{u}(t)) \geq 0, \quad \forall v \in D, t \in \Omega .
\end{aligned}
$$

Proof. Firstly we prove that for each fixed $u^{*}(t) \in D$, there exists a unique $\hat{u}(t) \in D, \hat{x}(t) \in A(t, \hat{u}(t)), \hat{y}(t) \in T(t, \hat{u}(t))$ such that

$$
\begin{gathered}
\langle N(\hat{x}(t), \hat{y}(t)), \eta(v, \hat{u}(t))\rangle+b\left(u^{*}(t), v\right)- \\
b\left(u^{*}(t), \hat{u}(t)\right) \geq 0, \quad \forall v \in D, t \in \Omega
\end{gathered}
$$

For any fixed $u^{*} \in D$, we define a function $\varphi$ : $\Omega \times D \times D \rightarrow(-\infty,+\infty]$ by $\varphi(t, v, u)=\langle N(x(t), y(t)), \eta(u, v)\rangle+b\left(u^{*}, u\right)-$ $b\left(u^{*}, v\right), \quad \forall v, u \in D, t \in \Omega$,

where $x(t) \in A(t, u), y(t) \in T(t, u)$.

Since $A$ and $T$ are the random multi-valued mappings induced by the random fuzzy mappings $\tilde{A}$ and $\tilde{T}$, respectively, i.e. for each $u \in D$, $A(\cdot, u)$ and $T(\cdot, u)$ are measurable mappings, so for any fixed $(v, u) \in D \times D, t \rightarrow \varphi(t, v, u)$ is measureable.

For any $v \in D$, the mapping $u \rightarrow \eta(u, v)$ is continuous. Then for each $v \in D$ and any sequence $\left\{u_{n}\right\} \subset D$ with $u_{n} \rightarrow u$, we have $\eta\left(u_{n}, v\right) \rightarrow \eta(u, v) \quad(n \rightarrow \infty)$. Since for each $t \in \Omega$, the mappings $A(t, \cdot), T(t, \cdot)$ are $\hat{H}-$ continuous, it follows for any fixed $(t, v) \in \Omega \times D$ that

$$
\begin{gathered}
\left|\left\langle N\left(x_{n}(t), y_{n}(t)\right), \eta\left(u_{n}, v\right)\right\rangle-\langle N(x(t), y(t)), \eta(u, v)\rangle\right| \\
\leq\left|\left\langle N\left(x_{n}(t), y_{n}(t)\right)-N(x(t), y(t)), \eta\left(u_{n}, v\right)\right\rangle\right|+ \\
\quad\left|\left\langle N(x(t), y(t)), \eta\left(u_{n}, v\right)-\eta(u, v)\right\rangle\right| \\
\leq\left|\left\langle N\left(x_{n}(t), y_{n}(t)\right)-N\left(x(t), y_{n}(t)\right), \eta\left(u_{n}, v\right)\right\rangle\right|+ \\
\quad\left|\left\langle N\left(x(t), y_{n}(t)\right)-N(x(t), y(t)), \eta\left(u_{n}, v\right)\right\rangle\right|+ \\
\quad\left|\left\langle N(x(t), y(t)), \eta\left(u_{n}, v\right)-\eta(u, v)\right\rangle\right| \\
\leq\left\|N\left(x_{n}(t), y_{n}(t)\right)-N\left(x(t), y_{n}(t)\right)\right\| \cdot\left\|\eta\left(u_{n}, v\right)\right\|+
\end{gathered}
$$

$$
\begin{aligned}
& \left\|N\left(x(t), y_{n}(t)\right)-N(x(t), y(t))\right\| \cdot\left\|\eta\left(u_{n}, v\right)\right\|+ \\
& \|N(x(t), y(t))\| \cdot\left\|\eta\left(u_{n}, v\right)-\eta(u, v)\right\| \\
& \leq k_{11}\left\|x_{n}(t)-x(t)\right\| \cdot\left\|\eta\left(u_{n}, v\right)\right\|+ \\
& k_{12}\left\|y_{n}(t)-y(t)\right\| \cdot\left\|\eta\left(u_{n}, v\right)\right\|+ \\
& \|N(x(t), y(t))\| \cdot\left\|\eta\left(u_{n}, v\right)-\eta(u, v)\right\| \\
& \leq k_{11} \lambda_{1}\left\|u_{n}-u\right\| \cdot\left\|\eta\left(u_{n}, v\right)\right\|+ \\
& k_{12} \lambda_{2}\left\|u_{n}-u\right\| \cdot\left\|\eta\left(u_{n}, v\right)\right\|+ \\
& \|N(x(t), y(t))\| \cdot\left\|\eta\left(u_{n}, v\right)-\eta(u, v)\right\| \\
& \rightarrow 0 \quad(n \rightarrow \infty) .
\end{aligned}
$$

Therefore for each fixed $(t, v) \in \Omega \times D$, the function $\quad u \rightarrow\langle N(x(t), y(t)), \eta(u, v)\rangle$ is continuous on $D$, where $x(t) \in A(t, u), y(t) \in$. $T(t, u)$. Since the function $u \rightarrow b\left(u^{*}, u\right)$ is continuous and convex on $D$ by the remark 2.1 (2), so for each fixed $(t, v) \in \Omega \times D, u \rightarrow \varphi(t, v, u)$ is continuous on $D$. Since the function $v \rightarrow$ $b\left(u^{*}, v\right)$ is continuous on $D$ and for any $u \in D$, $v \rightarrow \eta(u, v)$ is semicontinuous, so for each fixed $(t, u) \in \Omega \times D, v \rightarrow \varphi(t, v, u)$ is semicontinuous on $D$. Thus, we can confirm that the function $\varphi(t, v, u)$ satisfies the conditions (i)(ii)(iii) in Lemma 3.2 .

Now we prove that the function $\varphi(t, v, u)$ satisfies the condition (iv) in Lemma 3.2. We suppose that the function $\varphi(t, v, u)$ satisfies the condition (iv) of Lemma 3.2. If it is not true, there exists $t_{0} \in \Omega$, a finite set $\left\{v_{1}, v_{2}, \ldots, v_{m}\right\} \subset D$ and $u=\sum_{i=1}^{m} \lambda_{i} v_{i} \quad\left(\lambda_{i} \geq 0, \sum_{i=1}^{m} \lambda_{i}=1\right)$, such that $\varphi\left(t_{0}, v_{i}, u\right)>0$ for all $i=1,2, \ldots . m$, that is 
$\left\langle N\left(x\left(t_{0}\right), y\left(t_{0}\right)\right), \eta\left(u, v_{i}\right)\right\rangle+b\left(u^{*}, u\right)-b\left(u^{*}, v_{i}\right)>0$. It follows that

$$
\begin{gathered}
\sum_{i=1}^{m} \lambda_{i}\left\langle N\left(x\left(t_{0}\right), y\left(t_{0}\right)\right), \eta\left(u, v_{i}\right)\right\rangle+b\left(u^{*}, u\right)- \\
\sum_{i=1}^{m} \lambda_{i} b\left(u^{*}, v_{i}\right)>0
\end{gathered}
$$

Noting that $b(u, v)$ is convex in the second argument, that is

$$
\sum_{i=1}^{m} \lambda_{i} b\left(u^{*}, v_{i}\right) \geq b\left(u^{*}, \sum_{i=1}^{m} \lambda_{i} v_{i}\right)=b\left(u^{*}, u\right),
$$

we have

$$
\sum_{i=1}^{m} \lambda_{i}\left\langle N\left(x\left(t_{0}\right), y\left(t_{0}\right)\right), \eta\left(u, v_{i}\right)\right\rangle>0
$$

Since for any $t \in \Omega$, the mappings $u \rightarrow N(x(t)$, $y(t))$ and $\eta$ have the 0 -diagonally concave relation in $v$, so for any $t \in \Omega$,

$$
\sum_{i=1}^{m} \lambda_{i}\left\langle N(x(t), y(t)), \eta\left(u, v_{i}\right)\right\rangle \leq 0,
$$

which contradicts (3.2). Therefore, for any $t \in \Omega$, any finite set $\left\{v_{1}, v_{2}, \ldots, v_{m}\right\} \subset D$, and $u=\sum_{i=1}^{m} \lambda_{i} v_{i} \quad\left(\lambda_{i} \geq 0, \sum_{i=1}^{m} \lambda_{i}=1\right)$, we have $\varphi\left(t, v_{i}, u\right) \leq 0 \quad(i=1,2, \ldots m)$. Thus condition (iv) of lemma 3.2 holds.

For each $t \in \Omega$, let

$$
\begin{aligned}
& \theta=\frac{1}{k_{21}+k_{22}}\left(\alpha \cdot\left\|N\left(x^{*}(t), y^{*}(t)\right)\right\|+\gamma \cdot\left\|u^{*}\right\|\right), \\
& K=\left\{u \in D:\left\|u-u^{*}\right\| \leq \theta\right\}, D_{0}=\left\{u^{*}\right\},
\end{aligned}
$$

then $K$ and $D_{0}$ are both compact convex subsets of $D$. By assumptions (1) - (4) of the theorem, for each $u \in D \backslash K$, there exist $u^{*} \in \operatorname{Co}\left(D_{0} \cup\right.$ $\{u\}), x^{*}(t) \in A\left(t, u^{*}\right), y^{*}(t) \in T\left(t, u^{*}\right)$ such that $\varphi\left(t, u^{*}, u\right)=\left\langle N(x(t), y(t)), \eta\left(u, u^{*}\right)\right\rangle+b\left(u^{*}, u\right)-b\left(u^{*}, u^{*}\right)$ $=\left\langle N(x(t), y(t))-N\left(x^{*}(t), y(t)\right), \eta\left(u, u^{*}\right)\right\rangle+$ $\left\langle N\left(x^{*}(t), y(t)\right)-N\left(x^{*}(t), y^{*}(t)\right), \eta\left(u, u^{*}\right)\right\rangle+$ $\left\langle N\left(x^{*}(t), y^{*}(t)\right), \eta\left(u, u^{*}\right)\right\rangle+$ $b\left(u^{*}, u\right)-b\left(u^{*}, u^{*}\right)$

$$
\begin{gathered}
\geq k_{21}\left\|u-u^{*}\right\|^{2}+k_{22}\left\|u-u^{*}\right\|^{2}-\alpha\left\|N\left(x^{*}(t), y^{*}(t)\right)\right\| \cdot \\
\left\|u-u^{*}\right\|-\gamma\left\|u^{*}\right\| \cdot\left\|u-u^{*}\right\| \\
=\left\|u-u^{*}\right\| \cdot\left[\left(k_{21}+k_{22}\right)\left\|u-u^{*}\right\|-\right.
\end{gathered}
$$

$$
\left.\alpha\left\|N\left(x^{*}(t), y^{*}(t)\right)\right\|-\gamma\left\|u^{*}\right\|\right]>0 .
$$

Hence condition (5) of Lemma 3.2 is also satisfied. By Lemma 3.2, there exists a measurable mapping $u: \Omega \rightarrow D$, such that $\varphi(t, v, u(t)) \leq 0$ for all $v \in D$ and $t \in \Omega$.

We know the mapping $N(\cdot, \cdot)$ is Lipschitz continuous in first argument and in second argument, and the mappings $A(t, \cdot), T(t, \cdot)$ are $\hat{H}-$ continuous. Based on Lemma3.1, we obtain that for the measurable mapping $\hat{u}: \Omega \rightarrow D$, there exist $\hat{x}(t) \in A(t, \hat{u}(t)), \hat{y}(t) \in T(t, \hat{u}(t))$ such that $\langle N(\hat{x}(t), \hat{y}(t)), \eta(v, \hat{u}(t))\rangle+b\left(u^{*}(t), v\right)-b\left(u^{*}(t), \hat{u}(t)\right)$

$$
\leq 0 . \quad \forall v \in D, t \in \Omega
$$

By $\eta(u(t), v)=-\eta(v, u(t))$, we have

$\langle N(\hat{x}(t), \hat{y}(t)), \eta(\hat{u}(t), v)\rangle+b\left(u^{*}(t), \hat{u}(t)\right)-$ $b\left(u^{*}(t), v\right) \geq 0, \quad \forall v \in D, t \in \Omega$

This implies that for any $t \in \Omega$ and for each fixed measurable mapping $u^{*}(t) \in D$, the measurable mapping $\hat{u}: \Omega \rightarrow D, \quad \hat{x}(t) \in A(t, \hat{u}(t)), \hat{y}(t) \in$ $T(t, \hat{u}(t))$ is a random solution of the Auxiliary problem (3.1).

Now we prove that for any $t \in \Omega$, the measureable mapping $t \rightarrow \hat{u}(t), \hat{x}(t) \in A(t, \hat{u}(t)), \hat{y}(t) \in$ $T(t, \hat{u}(t))$ is a unique random solution of the auxiliary problem (3.1). Supposing the measurable mappings $u_{1}(t) \in D, x_{1}(t) \in A\left(t, u_{1}(t)\right), y_{1}(t) \in$ $T\left(t, u_{1}(t)\right)$ and $u_{2}(t) \in D, x_{2}(t) \in A\left(t, u_{2}(t)\right)$, $y_{2}(t) \in T\left(t, u_{2}(t)\right)$ are two random solutions of problem (3.1), we have the conclusion that for all $v \in D, t \in \Omega$,

$$
\begin{gathered}
\left\langle N\left(x_{1}(t), y_{1}(t)\right), \eta\left(v, u_{1}(t)\right)\right\rangle+ \\
b\left(u^{*}(t), v\right)-b\left(u^{*}(t), u_{1}(t)\right) \geq 0, \\
\left\langle N\left(x_{2}(t), y_{2}(t)\right), \eta\left(v, u_{2}(t)\right)\right\rangle+ \\
b\left(u^{*}(t), v\right)-b\left(u^{*}(t), u_{2}(t)\right) \geq 0
\end{gathered}
$$

Taking $v=u_{2}(t)$ in (3.3) and $v=u_{1}(t)$ in (3.4) and adding two inequalities, by the assumption on the function $b$, we obtain

$$
\begin{aligned}
& \left\langle N\left(x_{1}(t), y_{1}(t)\right), \eta\left(u_{2}(t), u_{1}(t)\right)\right\rangle+ \\
& \quad\left\langle N\left(x_{2}(t), y_{2}(t)\right), \eta\left(u_{1}(t), u_{2}(t)\right)\right\rangle \geq 0
\end{aligned}
$$

Since for all $u, v \in D, \eta(u, v)=-\eta(v, u)$, we have

$$
\left\langle N\left(x_{2}(t), y_{2}(t)\right)-N\left(x_{1}(t), y_{1}(t)\right), \eta\left(u_{2}(t), u_{1}(t)\right)\right\rangle
$$$$
\leq 0
$$

Noting that $N(\cdot, \cdot)$ is $\eta$-strongly monotone with respect to the random multi-valued mapping $A$ in first argument with constant $k_{21}>0$, and $\eta-$ strongly monotone with respect to the random multivalued mapping $T$ in second argument with constant $k_{22}>0$, we get 


$$
\begin{aligned}
& \left(k_{21}+k_{22}\right)\left\|u_{2}(t)-u_{1}(t)\right\|^{2} \\
\leq & \left\langle N\left(x_{2}(t), y_{2}(t)\right)-N\left(x_{1}(t), y_{2}(t)\right), \eta\left(u_{2}(t), u_{1}(t)\right)\right\rangle+ \\
& \left\langle N\left(x_{1}(t), y_{2}(t)\right)-N\left(x_{1}(t), y_{1}(t)\right), \eta\left(u_{2}(t), u_{1}(t)\right)\right\rangle
\end{aligned}
$$

$\leq 0$.

Since $k_{21}, k_{22}>0$, we have $u_{1}(t)=u_{2}(t)$.

Further, let

$$
x_{1}(t) \in A\left(t, u_{1}(t)\right), x_{2}(t) \in A\left(t, u_{2}(t)\right)
$$

and

$$
y_{1}(t) \in T\left(t, u_{1}(t)\right), y_{2}(t) \in T\left(t, u_{2}(t)\right),
$$

we have

$$
\begin{aligned}
\left\|x_{1}(t)-x_{2}(t)\right\| & \leq H\left(A\left(t, u_{1}(t)\right), A\left(t, u_{2}(t)\right)\right) \\
& \leq \lambda_{1}\left\|u_{1}(t)-u_{2}(t)\right\|, \\
\left\|y_{1}(t)-y_{2}(t)\right\| & \leq H\left(T\left(t, u_{1}(t)\right), T\left(t, u_{2}(t)\right)\right) \\
& \leq \lambda_{2}\left\|u_{1}(t)-u_{2}(t)\right\| .
\end{aligned}
$$

So we get $x_{1}(t)=x_{2}(t)$ and $y_{1}(t)=y_{2}(t)$, which imply that for any $t \in \Omega$ and the measurable mapping $u(t) \in D$, the mappings $\hat{u}(t) \in D$, $\hat{x}(t) \in A(t, \hat{u}(t)), \hat{y}(t) \in T(t, \hat{u}(t))$ (denote it by $w$ in the sequel) is a unique random solution of the auxiliary problem (3.1). Thus we have proved that for each $t \in \Omega$ and the measurable mapping $u(t) \in D$, there exists a unique solution $w$ satisfying (3.1). Defining a mapping $F: D \rightarrow D$ by $u^{*}(t) \rightarrow w\left(u^{\square}(t)\right)$, we will prove that the mapping $F$ is a contraction mapping. Indeed, for any $u_{1}^{*}(t), u_{2}^{*}(t) \in D$, there exist unique $W_{1}=$ $F\left(u_{1}^{*}(t)\right), w_{2}=F\left(u_{2}{ }^{*}(t)\right)$, for all $v \in D$ and $t \in \Omega$ such that

$$
\begin{gathered}
\left\langle N\left(x_{1}(t), y_{1}(t)\right), \eta\left(v, u_{1}(t)\right)\right\rangle+ \\
b\left(u_{1}^{*}(t), v\right)-b\left(u_{1}^{*}(t), u_{1}(t)\right) \geq 0, \\
\left\langle N\left(x_{2}(t), y_{2}(t)\right), \eta\left(v, u_{2}(t)\right)\right\rangle+ \\
b\left(u_{2}^{*}(t), v\right)-b\left(u_{2}^{*}(t), u_{2}(t)\right) \geq 0 .
\end{gathered}
$$

Taking $v=u_{2}(t)$ in (3.5) and $v=u_{1}(t)$ in (3.6) and adding two inequalities, we have

$$
\begin{aligned}
& \left\langle N\left(x_{1}(t), y_{1}(t)\right), \eta\left(u_{2}(t), u_{1}(t)\right)\right\rangle+ \\
& \left\langle N\left(x_{2}(t), y_{2}(t)\right), \eta\left(u_{1}(t), u_{2}(t)\right)\right\rangle+ \\
& b\left(u_{1}^{*}(t)-u_{2}{ }^{*}(\mathrm{t}), u_{2}(t)\right)-b\left(u_{1}^{*}(t)-u_{2}{ }^{*}(t),\right. \\
& \left.u_{1}(t)\right) \geq 0 .
\end{aligned}
$$

By $\eta(u, v)=-\eta(v, u)$ and the assumption on $b(\cdot, \cdot)$, we have

$$
\begin{aligned}
& \left(k_{21}+k_{22}\right)\left\|u_{2}(t)-u_{1}(t)\right\|^{2} \\
& \leq\left\langle N\left(x_{1}(t), y_{1}(t)\right)-N\left(x_{2}(t), y_{1}(t)\right), \eta\left(u_{1}(t), u_{2}(t)\right)\right\rangle+ \\
& \left\langle N\left(x_{2}(t), y_{1}(t)\right)-N\left(x_{2}(t), y_{2}(t)\right), \eta\left(u_{1}(t), u_{2}(t)\right)\right\rangle \\
& \leq b\left(u_{1}{ }^{*}(t)-u_{2}{ }^{*}(t), u_{2}(t)\right)-b\left(u_{1}{ }^{*}(t)-u_{2}{ }^{*}(t), u_{1}(t)\right)
\end{aligned}
$$

$\leq \gamma\left\|u_{1}^{*}(t)-u_{2}^{*}(t)\right\| \cdot\left\|u_{1}(t)-u_{2}(t)\right\|$, which derives

$$
\left\|u_{1}(t)-u_{2}(t)\right\| \leq \frac{\gamma}{k_{21}+k_{22}}\left\|u_{1}^{*}(t)-u_{2}{ }^{*}(t)\right\|,
$$

$$
\begin{aligned}
\left\|x_{1}(t)-x_{2}(t)\right\| \leq H & \left(A\left(t, u_{1}(t)\right), A\left(t, u_{2}(t)\right)\right) \\
& \leq \frac{\lambda_{1} \gamma}{k_{21}+k_{22}}\left\|u_{1}^{*}(t)-u_{2}{ }^{*}(t)\right\|,
\end{aligned}
$$

$$
\begin{aligned}
\left\|y_{1}(t)-y_{2}(t)\right\| & \leq H\left(T\left(t, u_{1}(t)\right), T\left(t, u_{2}(t)\right)\right) \\
& \leq \frac{\lambda_{2} \gamma}{k_{21}+k_{22}}\left\|u_{1}^{*}(t)-u_{2}^{*}(t)\right\| .
\end{aligned}
$$

The inequalities (3.7, (3.8) and (3.9) together with $\gamma \in\left(0, k_{21}+k_{22}\right)$ and $0<\lambda_{1}, \lambda_{2} \leq 1$ result in that $F$ is a contraction mapping. Hence, there exists a unique point $\hat{u}(t) \in D$ such that $\hat{u}(t)=F(\hat{u}(t))$ and

$$
\begin{gathered}
\langle N(\hat{x}(t), \hat{y}(t)), \eta(v, \hat{u}(t))\rangle+b(\hat{u}(t), v)- \\
b(\hat{u}(t), \hat{u}(t)) \geq 0, \quad \forall v \in D, t \in \Omega
\end{gathered}
$$

Now

we know

$\hat{u}(t) \in D, \hat{x}(t) \in A(t, \hat{u}(t)), \hat{y}(t) \in T(t, \hat{u}(t))$

is the unique solution of the problem (2.1).

This completes the proof.

\section{References}

[1] M.A. Noor, Some recent advances in variational inequalities Part I: basic concepts, New Zealand J. Math. , 26:53 - 80, 1997.

[2] M.A. Noor, Some recent advances in variational inequalities Part II: other concepts, New Zealand J. Math. , 26:229 - 255, 1997.

[3] G. Tian, Generalized quasi variational-like inequality problem, Math. Oper. Res. , 18:752-764, 1993.

[4] J. C. Yao, The generalized quasi variational inequality problem with applications, J. Math. Anal. Appl. 158 :139-160, 1991.

[5] P. D. Panagiotopoulos and G. E. Stavroulakis, New types of variational principles based on the notion of quasidifferentiability, Acta Mech, 94: 171-194, 1992.

[6] P. Cubiotti, Existence of solutions for lower semicontinuous quasi equilibrium problems, comput. Math. Appl. , 30: 11-22, 1995.

[7] R. Glowinski, J. L. Lions, and R. Tremolieres, Numerical analysis of variational inequalities, North-Holland, Amsterdam, 1981.

[8] M. Lassonde, On the use of KKM 
multifunction in .xed point theory and related topics , J. Math. Anal. Appl. , 97: 151 - 201, 1983 .

[9] N.J. Huang and C.X Deng, Auxiliary principle and iterative algorithms for generalized set-valued strongly nonlinear mixed variational-like inequalities, J. Math. Anal.Appl. , 256:345-359, 2001.

[10]X.P. Ding, Existence and Algorithm of Solutions for Nonlinear Mixed Variational-like Inequalities in Banach spaces, J. Comput. Appl. Math., 157: 419-434, 2003 .

[11]S.S. Chang, Y. Zhu, On variational inequalities for fuzzy mappings, Fuzzy Set. Syst. 32:359 - 367, 1989.

[12]M.H. Shih, K.K. Tan, Generalized quasi-variational inequalities in locally convex spaces, J. Math. Anal. Appl. ,108: 333 - 343, 1985.

[13] R. Ahmad, F. F. Bazan, An iterative algorithm for random generalized nonlinear mixed variational inclusions for random fuzzy mappings, Appl. Math. Comput., 167:1400 1411, 2005.

[14] N.J. Huang, Random generalized nonlinear variational inclusions for random fuzzy mappings, Fuzzy Set. Syst. ,105:437 444,1999.

[15] S.S. Chang, N.J. Huang, Random generalized set-valued quasi-complementarity problems, Acta Math. Appl. Sinica , 16: 396 - 405, 1993.

[16]T. Husain, E. Tarafdar, X.Z. Yuan, "Some results on random generalized games and random quasi-variational inequalities, Far East. J. Math. Sci. , 2: 35 - 55, 1994.

[17] N.J. Huang, Random generalized set-valued implicit variational inequalities, J. Liaoning Normal Univ. , 18: 89 - 93, 1995.

[18] X.P.Ding, General Algorithms of Solutions for Nonlinear Variational-like Inequalities in Reflexive Banach Spaces, Indian.J.Pure.Appl.Math.,29(2): $\quad$ 109-120, 1998.

[19] X.H.Chen, Y.F.Liu, On the Generalized Nonlinear Variational-like Inequalities in Reflexive Banach Spaces, J.Nanjing.Univ.Math.Biquart. ,18(1): 96-103, 2001.

[20]X.P. Ding, Existence and Uniqueness of solutions for Random Mixed Variational-like Inequalities in Banach spaces, J. Sichuan Normal. University (Natural Science). , 20: 1-5, 1997.

[21] S.S. Chang, Fixed Point Theory with Applications, Chongqing Publishing House, Chongqing, 1984. 